\title{
Component-Mode-Based Monte Carlo Simulation for Efficient Probabilistic Vibration Analysis
}

\author{
Soo-Yeol Lee* and Matthew P. Castanier ${ }^{\dagger}$ \\ The University of Michigan, Ann Arbor, Michigan 48109-2125 \\ Christophe Pierre $\ddagger$ \\ McGill University, Montreal, Quebec H3A 2K6, Canada
}

\begin{abstract}
In this paper, component-mode-based implementations of Monte Carlo simulation (MCS) are presented for efficient probabilistic vibration analysis of complex structures with parameter uncertainties. First, a substructuring technique is used to generate reduced-order models of low- to mid-frequency vibration and power flow. The reduced-order model (ROM) is generated using component mode synthesis of finite element models, followed by a secondary modal analysis to reduce the number of degrees of freedom associated with the substructure interfaces. This formulation allows for efficient and accurate prediction of vibration transmission in complex structural systems. Then, the response is approximated over an uncertainty space to study the statistics of the power flow for a structure with parametric uncertainties. Two MCS techniques that employ first-order approximations of the ROMs are presented: (1) a nominal-ROM-based MCS for a case with many component-mode interactions over the uncertainty space, and (2) a mode-based MCS for a case with a few component-mode interactions. An iterative maximum search procedure is presented, which is used to find the upper bound of the power flow at a specific frequency. For the second MCS approach, this iterative procedure is also extended to yield various statistical properties of the response in addition to the upper bound. These statistical approximations of vibration power flow are demonstrated for two example structures, a reinforced L-shaped plate and a stiffened plate.
\end{abstract}

\section{Introduction}

Manufactured product always has some parameter uncertainties due to tolerances, material property A variations, in-service degradation, etc. The variability that is inherent in manufacturing and assembling parts produces a consequent variability in the dynamic response, and the vibration of a complex structure can be especially sensitive to such parameter variations. Thus, before manufacturing the product, it is desirable to be able to predict the variability of the dynamic behavior of the structure.

A general statistical approach based on finite element (FE) modeling is to use the Monte Carlo simulation technique to evaluate the statistical characteristics of the vibration response. While this approach is versatile and accurate, it may incur prohibitive computational cost for complex structures. To develop a more efficient approach, three steps can be considered as follows: (1) generate a reduced-order model (ROM) for a deterministic analysis, (2) employ a simplified model over an uncertainty space, and (3) apply a probabilistic analysis method.

Regarding the first step, various methods for generating a ROM for structural vibration analysis, based on a parent FE model, have been developed using substructuring techniques. ${ }^{1-5}$ This type of ROM provides a convenient framework for both optimization and probabilistic analysis with respect to parametric uncertainties. Regarding the second step, the probabilistic analysis can be expedited by using a simplified model over an uncertainty space (e.g., a response surface method, ${ }^{6,7}$ a perturbation method, ${ }^{8,9}$ etc.). Finally, a

${ }^{*}$ Graduate Student Research Assistant, Department of Mechanical Engineering. Student Member of AIAA.

${ }^{\dagger}$ Associate Research Scientist, Department of Mechanical Engineering. Senior Member of AIAA.

$\ddagger$ Dean, Faculty of Engineering. Senior Member of AIAA. 
specific probabilistic analysis method needs to be applied. Some choices are summarized as follows: (1) sampling methods, such as a standard Monte Carlo method ${ }^{10}$ and various Latin Hypercube designs; ${ }^{11,12}$ (2) most probable point (MPP)-based methods, such as the advanced mean value (AMV) method, ${ }^{13,14}$ the hybrid mean value (HMV) method, ${ }^{15}$ and the sequential MPP search method; ${ }^{16,17}$ and (3) hybrid sampling methods utilizing the MPP, such as an importance sampling method, ${ }^{18}$ a radius-based importance sampling method, ${ }^{19}$ or an adaptive importance sampling method. ${ }^{20}$ The objective of this paper is to present an efficient technique using a simplified model integrated with the ROM over an uncertainty space, in order to expedite a probabilistic vibration analysis.

In order to obtain a simplified mathematical model, the response surface method has been extensively studied and utilized for optimization, reliability analysis, and probabilistic analysis. It is known that the response surface method depends on the locations and numbers of the sampling points, and it requires that one ROM be constructed for each sampling point over an uncertainty space. To alleviate the sensitivity of the positions of the sampling points around the resonances of the structural vibration, a locally linear interpolation (LLI) method has been proposed, ${ }^{17,21}$ and it was shown to have greater efficiency with less sensitivity of the positions of the sampling points compared to a response surface method employing the radial based function. ${ }^{17,21}$ However, it was also found that the efficiency of the LLI method decreases as the number of random variables increases. As for the perturbation method, it has been widely studied and employed for cases where a random system equation is replaced with a theoretically infinite number of deterministic system equations. In reality, there is a restriction of the small variance of the uncertainties for a few terms of the infinite series.

In this paper, vibration power flow analysis based on component mode synthesis (CMS) and characteristic constraint (CC) modes ${ }^{5,22-24}$ is applied to study structures with uncertainties. The finite element (FE) model of the full structure is divided into separate component FE models, and a fixed-interface CMS approach ${ }^{1}$ is adopted. Then, secondary modal analysis is used to further reduce the size of the CMS model. For the purpose of performing power flow analysis, this secondary modal analysis is applied to the interfaces between components. More specifically, an eigenanalysis is performed on the constraint mode partitions of the CMS mass and stiffness matrices, yielding the $\mathrm{CC}$ modes. The $\mathrm{CC}$ modes may then be selected to reduce the degrees of freedom (DOF) for the component interfaces, yielding a reduced-order model (ROM). Thus, a deterministic vibration analysis can be done efficiently with the ROM. Then, for probabilistic vibration analysis, two simplified mathematical models are constructed over an uncertainty space and integrated with a Monte Carlo simulation, yielding statistical properties of vibration power flow. The first approach utilizes a first-order approximation of a full ROM system, and it is called a nominal-ROM-based MCS method. The second approach utilizes a first-order approximation of a ROM system that is diagonalized based on a modal analysis, and the uncertainty space is searched in an iterative manner for the resonance points. This second approach is called a mode-based MCS method.

The contributions of this paper are summarized as follows. First, two first-order approximations of a ROM - using a full ROM system and a diagonalized ROM system - are combined with Monte Carlo simulation to perform probabilistic vibration analysis, and the advantages of each method are demonstrated. Second, an iterative maximum search procedure is presented that provides the upper bound of the vibration response at a specific frequency. Third, the iterative maximum search procedure is extended to the modebased MCS method, which can yield the statistical properties as well as the upper bound of the response.

This paper is organized as follows. First, the CC-mode-based reduced-order modeling and power flow analysis techniques are reviewed. Then, the nominal-ROM-based and mode-based MCS methods are described and validated with example structures. Conclusions are given in the final section of the paper.

\section{Background}

\section{A. Reduced-Order Modeling Based on Characteristic Constraint (CC) Modes}

The reduced-order modeling method based on characteristic constraint modes was presented in Ref. 5, and it is briefly described here. The mass and stiffness matrices from each substructure are first partitioned into the interior submatrices, the interface submatrices, and their coupling submatrices. Then, the Craig-Bampton method of $\mathrm{CMS}^{1}$ is applied to these substructure matrices, resulting in the mass, stiffness, and force matrices 
in the constraint-mode degrees of freedom (DOF) and normal-mode DOF as follows:

$$
\mathbf{M}_{i}=\left[\begin{array}{ll}
\mathbf{m}_{i}^{C} & \mathbf{m}_{i}^{C N} \\
\mathbf{m}_{i}^{C N^{T}} & \mathbf{m}_{i}^{N}
\end{array}\right] \quad \mathbf{K}_{i}=\left[\begin{array}{ll}
\mathbf{k}_{i}^{C} & \mathbf{0} \\
\mathbf{0} & \mathbf{k}_{i}^{N}
\end{array}\right] \quad \mathbf{f}_{i}=\left[\begin{array}{l}
\mathbf{f}_{i}^{C} \\
\mathbf{f}_{i}^{N}
\end{array}\right]
$$

where the subscript $i$ refers to each substructure. With the matrices Eq. (1) for individual submatrices, a CMS model of the global structure is constructed, and the synthesized CMS mass matrix may be written as

$$
\mathbf{M}^{C M S}=\left[\begin{array}{ccccc}
\overline{\mathbf{m}}^{C} & \mathbf{m}_{1}^{C N} & \mathbf{m}_{2}^{C N} & \cdots & \mathbf{m}_{n^{s s}}^{C N} \\
\mathbf{m}_{1}^{C N^{T}} & \mathbf{m}_{1}^{N} & \mathbf{0} & \cdots & \mathbf{0} \\
\mathbf{m}_{2}^{C N^{T}} & \mathbf{0} & \mathbf{m}_{2}^{N} & \cdots & \mathbf{0} \\
\vdots & \vdots & \vdots & \ddots & \vdots \\
\mathbf{m}_{n^{s s}}^{C N^{T}} & \mathbf{0} & \mathbf{0} & \cdots & \mathbf{m}_{n^{s s}}^{N}
\end{array}\right]
$$

where $n^{s s}$ is the number of substructures contained in the global structure. The component modal matrices, $\mathbf{m}_{i}^{N}$ and $\mathbf{k}_{i}^{N}$, are diagonalized and their size equals the number of vibration modes selected for the $i$ th substructure. However, the number of constraint-mode DOF, or the size of $\overline{\mathbf{m}}^{C}$ and $\overline{\mathbf{k}}^{C}$, is equal to the number of interface DOF in the FE model. Therefore, while the component partitions are, in general, greatly reduced relative to the parent FE model, there is no reduction for the interface DOF. For large-scale models of a vehicle structure, the size of the CMS model may be dominated by the interface DOF. Therefore, to obtain further order reduction, a secondary modal analysis is performed on the constraint-mode DOF:

$$
\overline{\mathbf{k}}^{C} \boldsymbol{\psi}_{n}=\lambda_{n} \overline{\mathbf{m}}^{C} \boldsymbol{\psi}_{n}
$$

These eigenvectors, $\boldsymbol{\psi}_{n}$, may be expressed in finite element coordinates for component structure $i$ using the following transformation

$$
\boldsymbol{\Phi}_{i}^{C C}=\boldsymbol{\Phi}_{i}^{C} \boldsymbol{\beta}_{i}^{C} \boldsymbol{\Psi}
$$

where $\boldsymbol{\Psi}=\left[\boldsymbol{\psi}_{1} \boldsymbol{\psi}_{2} \cdots \boldsymbol{\psi}_{n^{c c}}\right]$ is a selected set of the interface eigenvectors with $n^{c c}$ indicating the number of selected eigenvectors, and $\boldsymbol{\beta}_{i}^{C}$ is the matrix that maps the global interface DOF, $\mathbf{v}^{C}$, to the local DOF, $\mathbf{v}_{i}^{C}$. The vectors $\boldsymbol{\Phi}_{i}^{C C}$ are referred to as the characteristic constraint (CC) modes, because they are eigenvectorbased linear combinations of constraint modes that provide a set of characteristic displacement shapes for the interface.

The CMS model can now be transformed to a reduced-order model (ROM) by selecting a truncated set of $\mathrm{CC}$ modes for the frequency range of interest, and then using these vectors to project the CMS matrices onto the reduced basis. The equations of motion of the ROM can be expressed by

$$
\frac{1}{j \omega}\left[-\omega^{2} \mathbf{M}^{R O M}+(1+j \eta) \mathbf{K}^{R O M}\right] \mathbf{v}^{R O M}=\mathbf{f}^{R O M}
$$

where $\eta$ is the structural damping factor and $j=\sqrt{-1}$. The mass matrix, $\mathbf{M}^{R O M}$ can be explicitly written as

where

$$
\mathbf{M}^{R O M}=\left[\begin{array}{ccccc}
\overline{\mathbf{m}}^{C C} & \overline{\mathbf{m}}_{1}^{C N} & \overline{\mathbf{m}}_{2}^{C N} & \cdots & \overline{\mathbf{m}}_{n^{s s}}^{C N} \\
\overline{\mathbf{m}}_{1}^{C N^{T}} & \mathbf{m}_{1}^{N} & \mathbf{0} & \cdots & \mathbf{0} \\
\overline{\mathbf{m}}_{2}^{C N^{T}} & \mathbf{0} & \mathbf{m}_{2}^{N} & & \mathbf{0} \\
\vdots & \vdots & & \ddots & \vdots \\
\overline{\mathbf{m}}_{n^{s s}}^{C N^{T}} & \mathbf{0} & \mathbf{0} & \cdots & \mathbf{m}_{n^{s s}}^{N}
\end{array}\right]
$$

Now, every matrix partition has been reduced by some form of modal analysis. It should be noted that the ROM can be used not only to calculate the vibration response, but also the vibration energy that is transmitted between component structures, which is referred to as the power flow. Using power flow analysis, the energy level of the substructures can be calculated, and the paths of vibration transmission from the source to key response points can be identified. 


\section{B. Power Flow Analysis}

The power flow analysis requires first solving the system equations of the ROM, Eq. (5). For a time-harmonic excitation, the power flowing out of substructure $i$ at the discrete frequency $\omega$ through a specific interface with substructure $l$ can be described with the traction force vector $\mathbf{t}_{i}$ and the corresponding velocity vector on the interface $\mathbf{v}_{i}^{C}$ as

$$
g_{i l}(\omega)=-\frac{1}{2} \operatorname{Re}\left[\mathbf{v}_{i}^{C}(\omega)^{*} \boldsymbol{\alpha}_{i l}^{T} \boldsymbol{\alpha}_{i l} \mathbf{t}_{i}(\omega)\right]
$$

where $\boldsymbol{\alpha}_{i l}$ is a matrix mapping the total interface DOF of substructure $i$ to the interface DOF between substructure $i$ and $l$. From component equations of motion, the traction force vector $\mathbf{t}_{i}$ can be formulated in terms of $\mathbf{v}_{i}^{C}$ as

$$
\mathbf{t}_{i}=\mathbf{Z}_{i} \mathbf{v}_{i}^{C}-\mathbf{F}_{i}
$$

where

$$
\begin{gathered}
\mathbf{Z}_{i}=\mathbf{z}_{i}^{C}+\omega^{2} \mathbf{m}_{i}^{C N} \mathbf{z}_{i}^{N-1} \mathbf{m}_{i}^{C N^{T}} \\
\mathbf{F}_{i}=\mathbf{f}_{i}^{C}-j \omega \mathbf{m}_{i}^{C N} \mathbf{z}_{i}^{N-1} \mathbf{f}_{i}^{N}
\end{gathered}
$$

where $\mathbf{z}_{i}^{C}$ is the impedance matrix of substructure $i$ associated with constraint modes, and $\mathbf{z}_{i}^{N}$ the impedance matrix of substructure $i$ associated with component normal modes. The term, $\operatorname{Re}\left[\mathbf{v}_{i}^{C}{ }^{*} \boldsymbol{\alpha}_{i l}^{T} \boldsymbol{\alpha}_{i l} \mathbf{t}_{i}\right]$, in Eq. (8) can be estimated using Eq. (9)

$$
\operatorname{Re}\left[\mathbf{v}_{i}^{C^{*}} \boldsymbol{\alpha}_{i l}^{T} \boldsymbol{\alpha}_{i l} \mathbf{t}_{i}\right]=\mathbf{v}_{i}^{C^{*}} \operatorname{Re}\left[\mathbf{A}_{i l} \mathbf{Z}_{i}\right] \mathbf{v}_{i}^{C}-\operatorname{Re}\left[\mathbf{v}_{i}^{C^{*}} \mathbf{A}_{i l} \mathbf{F}_{i}\right]
$$

where $\mathbf{A}_{i l}=\boldsymbol{\alpha}_{i l}^{T} \boldsymbol{\alpha}_{i l}$, which is a diagonal matrix with only 0 and 1 in its diagonal elements. Furthermore, the computational efficiency can be achieved by projecting $\mathbf{t}_{i}$ and $\mathbf{v}_{i}^{C}$ onto CC modes. The right-hand side of Eq. (12) is now expressed in terms of CC-mode velocities, $\mathbf{v}^{C C}$, in place of the constraint-mode velocities, $\mathbf{v}^{C}$ :

$$
\operatorname{Re}\left[\mathbf{v}_{i}^{C *} \boldsymbol{\alpha}_{i l}^{T} \boldsymbol{\alpha}_{i l} \mathbf{t}_{i}\right]=\mathbf{v}^{C C^{*}} \widehat{\mathbf{C}}_{i l} \mathbf{v}^{C C}-\operatorname{Re}\left[\mathbf{v}^{C C^{*}} \widehat{\mathbf{F}}_{i l}\right]
$$

where

$$
\begin{gathered}
\widehat{\mathbf{C}}_{i l}=\boldsymbol{\Psi}^{T} \boldsymbol{\beta}_{i}^{C^{T}} \operatorname{Re}\left[\mathbf{A}_{i l} \mathbf{Z}_{i}\right] \boldsymbol{\beta}_{i}^{C} \boldsymbol{\Psi} \\
\widehat{\mathbf{F}}_{i l}=\boldsymbol{\Psi}^{T} \boldsymbol{\beta}_{i}^{C^{T}} \mathbf{A}_{i l} \mathbf{F}_{i}
\end{gathered}
$$

Equation (13) can be evaluated by solving CC-mode velocities, $\mathbf{v}^{C C}$, from Eq. (5), in which $\mathbf{v}^{C C}$ can be explicitly expressed as

$$
\mathbf{v}^{C C}=\left(\sum_{i=1}^{n^{s s}} \boldsymbol{\Psi}^{T} \boldsymbol{\beta}_{i}^{C^{T}} \mathbf{Z}_{i} \boldsymbol{\beta}_{i}^{C} \boldsymbol{\Psi}\right)^{-1}\left(\sum_{i=1}^{n^{s s}} \boldsymbol{\Psi}^{T} \boldsymbol{\beta}_{i}^{C^{T}} \mathbf{F}_{i}\right)
$$

Finally, the power flow can be expressed in the CC-mode coordinates of substructure $i$ as

$$
g_{i l}(\omega)=\frac{1}{2} \operatorname{Re}\left[\mathbf{v}^{C C^{*}} \widehat{\mathbf{F}}_{i l}\right]-\frac{1}{2} \mathbf{v}^{C C^{*}} \widehat{\mathbf{C}}_{i l} \mathbf{v}^{C C}
$$

\section{Probabilistic Power Flow Analysis}

For the probabilistic power flow analysis, a performance function given in Eq. (17) is utilized in this paper. Given the joint probability density function $(\mathrm{PDF}) f_{\mathbf{x}}(\mathbf{x})$ of random variables $\mathbf{x}$, the probability of the power flow can be expressed as

$$
\mathrm{P}\left[g(\mathbf{x} ; \omega) \leq g_{o}\right]=\iint \cdots \int_{g(\mathbf{x} ; \omega) \leq g_{o}} f_{\mathbf{x}}(\mathbf{x}) d \mathbf{x}
$$

It is a fundamental but difficult problem in probabilistic structural analysis to compute Eq. (18). With an implicit performance function, the multi-dimensional numerical integration can be extremely time-consuming. To evaluate Eq. (18) efficiently, probabilistic methods can be employed. In some cases, for more efficiency, a simplified mathematical model of $g(\mathbf{x} ; \omega)$ over an uncertainty space, such as a response surface method or a perturbation method, can also be utilized along with a probabilistic analysis method as shown in Fig. 1. In this paper, the use of a simplified mathematical model, which is integrated with a Monte Carlo simulation (MCS), will be examined. 


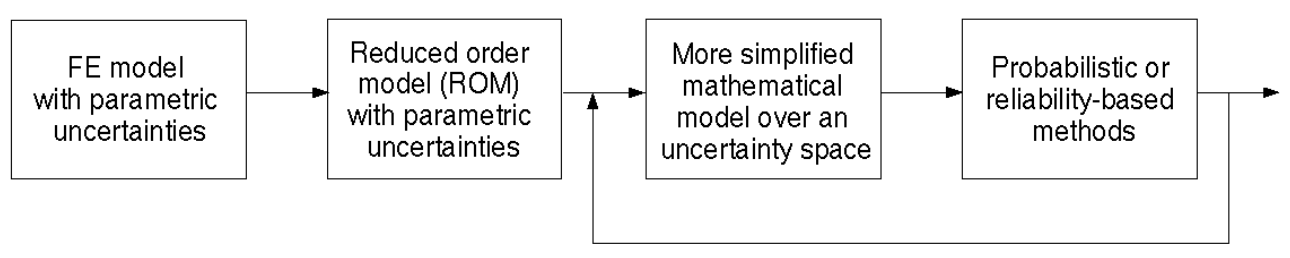

Figure 1. Flow chart for probabilistic vibration analysis of a structure with parametric uncertainties

\section{Method 1: Nominal-ROM-Based MCS}

\section{A. First-Order Approximation of Full ROM System}

In order to study the effect of parametric uncertainties, the component-based approach and reduced-order modeling technique described in the previous section are utilized. First, the uncertainties or design variables of interest are chosen and the system is substructured such that a parametric uncertainty can affect a whole substructure. Thus, fixed-interface normal modes for a nominal component can be used to capture the effects of parametric uncertainties. With a vector of random variables, $\mathbf{x}=\left(x_{1}, x_{2}, \cdots, x_{n}\right)$, the impedance and force matrices in ROM coordinates can be approximated over an uncertainty space as

$$
\begin{aligned}
\mathbf{Z}^{R O M}(\mathbf{x} ; \omega) & =\mathbf{Z}_{o}^{R O M}+\delta \mathbf{Z}^{R O M} \\
& =\mathbf{Z}^{R O M}(\mathbf{0} ; \omega)+\sum_{i=1}^{n}\left(\frac{\partial \mathbf{Z}^{R O M}}{\partial x_{i}}\right)_{\mathbf{x}=\mathbf{0}}\left(x_{i}-0\right) \\
\mathbf{f}^{R O M}(\mathbf{x} ; \omega) & =\mathbf{f}_{o}^{R O M}+\delta \mathbf{f}^{R O M} \\
& =\mathbf{f}^{R O M}(\mathbf{0} ; \omega)+\sum_{i=1}^{n}\left(\frac{\partial \mathbf{f}^{R O M}}{\partial x_{i}}\right)_{\mathbf{x}=\mathbf{0}}\left(x_{i}-0\right)
\end{aligned}
$$

In addition to $\mathbf{Z}^{R O M}$ and $\mathbf{f}^{R O M}$, the constraint modes $\boldsymbol{\Phi}_{i}^{C}$ required for calculating the forced response can also be approximated as

$$
\mathbf{\Phi}_{i}^{C}(\mathbf{x})=\boldsymbol{\Phi}_{i}^{C}(\mathbf{0})+\sum_{i=1}^{n}\left(\frac{\partial \boldsymbol{\Phi}_{i}^{C}}{\partial x_{i}}\right)_{\mathbf{x}=\mathbf{0}}\left(x_{i}-0\right)
$$

Then, with the approximations of Eq. (19), $\mathbf{v}^{R O M}$ is obtained directly from

$$
\mathbf{v}^{R O M}=\left(\mathbf{Z}_{o}^{R O M}+\delta \mathbf{Z}^{R O M}\right)^{-1}\left(\mathbf{f}_{o}^{R O M}+\delta \mathbf{f}^{R O M}\right)
$$

In this manner, the power flow or forced response can also be approximated over an uncertainty space, and a Monte Carlo simulation (MCS) can be performed efficiently. This is referred to as a nominal-ROM-based MCS method.

\section{B. Example Model 1: Reinforced L-shaped Plate}

To illustrate the application of the first-order approximation of the full ROM system, the reinforced L-shaped plate shown in Fig. 2 is used as an example. The system has free boundary conditions on all sides. The three component plates are referred to as plate 1 , plate 2 , and plate 3 . The dimensions of the plates are $0.55 \mathrm{~m} \times$ $1.6 \mathrm{~m}$ for plate $1,0.47 \mathrm{~m} \times 1.6 \mathrm{~m}$ for plate 2 , and $0.55 \mathrm{~m} \times 0.47 \mathrm{~m}$ for plate 3 . The material properties are Young's modulus $E=200 \mathrm{GPa}$, Poisson's ratio $\nu=0.3$, density $\rho=7827 \mathrm{~kg} / \mathrm{m}^{3}$, and thickness $t=10.16$ $\mathrm{mm}$. A finite element model of this plate was constructed using NASTRAN quadrilateral plate elements, and the mesh is shown in Fig. 2. The FE model has 4,752 DOF. The excitation for this example system is applied at two end points of plate 2 at its free edge, as illustrated in Fig. 2. The applied forces are sinusoidal, and their phases are determined randomly. 
A case of uncertainty is considered for the reinforced L-shaped plate. The case is that of two uncertain parameters, the thicknesses of plates 1 and 2, which have uniform distributions ranging from $-10 \%$ to $+10 \%$ about the nominal value. More specifically, the net vibration power flowing into plate 1 at $522.6 \mathrm{~Hz}$ is of interest. At this frequency, the response surface is constructed with the exact ROMs at $41 \times 41$ sampling points chosen to have equal interval over an uncertainty space, as shown in Fig. 3, in order to illustrate the complexity of the response surface. Thus, due to the complexity of the peaks shown in Fig. 3, it may not be efficient or accurate to construct the response surface with a few sampling points, and it is likely that such a response surface would not capture the vibration response peaks over an uncertainty space. Furthermore, the probabilistic analysis methods based on

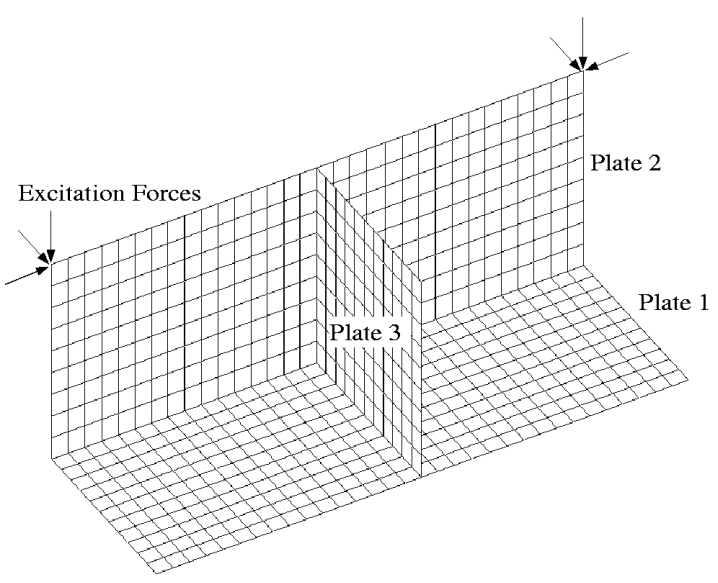

Figure 2. FE mesh for example model 1 (AMV) method, ${ }^{13}$ the hybrid mean value (HMV) method, ${ }^{15}$ or the sequential MPP search method ${ }^{16}$ are not well suited to handle this case. Therefore, Monte Carlo simulation (MCS) based on a first-order approximation of the full ROM system is proposed as an efficient method for probabilistic vibration analysis.

Figure 4 shows the CDF from the MCS with 2,000 exact ROMs, as well as from the MCS with 2,000 first-order approximations of full ROMs. Excellent agreement is seen for the CDF result. To show the efficiency of the first-order approximation of full ROM system, the CPU times are also compared in Table 1. For this case, it is clearly more efficient to utilize the first-order approximations of the full ROM system.

Table 1. Computational cost of MCS with 2,000 realizations for example model 1 at $522.58 \mathrm{~Hz}$

\begin{tabular}{|c|r|}
\hline Method & CPU Time \\
\hline Reference: MCS with exact ROMs & $929 \mathrm{~min}$ \\
Method 1: Nominal-ROM-Based MCS & $56 \mathrm{~min}$ \\
\hline
\end{tabular}

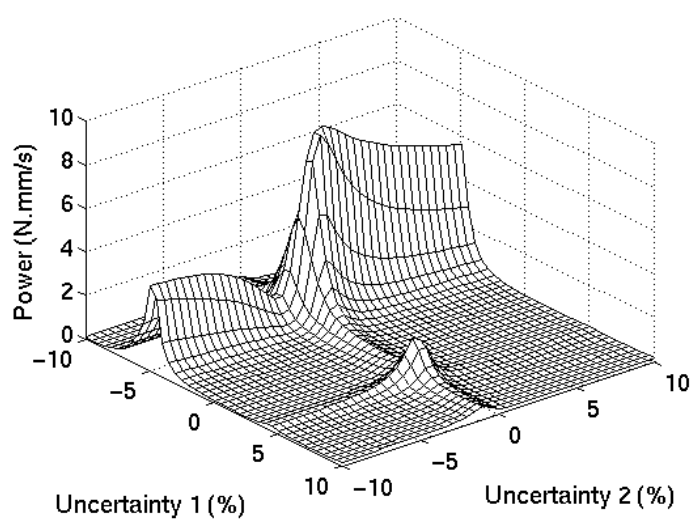

Figure 3. The response surface constructed for example model 1 at a specific frequency

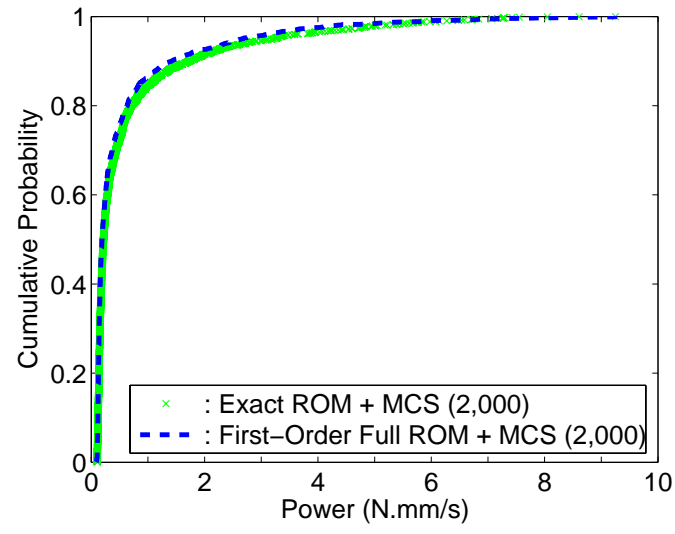

Figure 4. The cumulative probability from MCS using exact ROMs and MCS using a first-order approximation of the full ROM at a nominal point (nominal-ROM-based MCS method) 


\section{Method 2: Mode-Based MCS}

The approach described in the preceding section can alleviate the costs associated with constructing $\mathbf{Z}^{R O M}$ and $\mathbf{f}^{R O M}$ from the mass and stiffness matrices in FE coordinates. However, when the size of ROM is large, the computational cost for $\left(\mathbf{Z}_{o}^{R O M}+\delta \mathbf{Z}^{R O M}\right)^{-1}$ in Eq. (21) becomes more expensive. Therefore, an alternative first-order approximation approach is proposed in this section.

\section{A. First-Order Approximation of Diagonalized ROM System}

In order to obtain the first-order approximation of the diagonalized ROM about a key selected sampling point, or center point, in the parameter space, an eigenanalysis can be performed

$$
\mathbf{K}^{R O M} \boldsymbol{\Gamma}=\mathbf{M}^{R O M} \mathbf{\Gamma} \boldsymbol{\Lambda}
$$

where $\mathbf{K}^{R O M}$ and $\mathbf{M}^{R O M}$ are stiffness and mass matrices for the ROM at the center point, and $\boldsymbol{\Lambda}$ is a diagonal matrix of the free vibration natural frequencies of the whole complex structure. Then, the eigenmatrix $\boldsymbol{\Gamma}$ can be partitioned as

$$
\boldsymbol{\Gamma}=\left[\gamma^{C C^{T}} \gamma_{1}^{N^{T}} \gamma_{2}^{N^{T}} \cdots \gamma_{n^{s s}}^{N^{T}}\right]^{T}
$$

and the equations of motion of the ROM can be diagonalized:

$$
\frac{1}{j \omega}\left[-\omega^{2} \overline{\mathbf{M}}+(1+j \eta) \overline{\mathbf{K}}\right] \overline{\mathbf{v}}=\overline{\mathbf{f}}
$$

where

$$
\overline{\mathbf{M}}=\boldsymbol{\Gamma}^{T} \mathbf{M}^{R O M} \boldsymbol{\Gamma}, \quad \overline{\mathbf{K}}=\boldsymbol{\Gamma}^{T} \mathbf{K}^{R O M} \boldsymbol{\Gamma}, \quad \overline{\mathbf{f}}=\boldsymbol{\Gamma}^{T} \mathbf{f}^{R O M}
$$

and

$$
\mathbf{v}^{R O M}=\mathbf{\Gamma} \overline{\mathbf{v}}
$$

The matrices of $\overline{\mathbf{M}}$ and $\overline{\mathbf{K}}$ are diagonalized by the projection onto the global modes of the ROM, and the impedance matrix $\overline{\mathbf{Z}}$ can be expressed with $\overline{\mathbf{M}}$ and $\overline{\mathbf{K}}$ as

$$
\overline{\mathbf{Z}}=\frac{1}{j \omega}\left[-\omega^{2} \overline{\mathbf{M}}+(1+j \eta) \overline{\mathbf{K}}\right]
$$

Assume that the global mode shapes are approximately the same over some subdomain of the parameter space, or uncertainty range, around each center point. Then, the key process is to identify each center point and the corresponding uncertainty range. This approach is composed of six steps, which are summarized as follows:

STEP 1: A set $S$ having reference sampling points $\mathbf{x}_{r}$ is defined such that reference sampling points have uniform distribution over an uncertainty range of interest, as shown in Fig. 5. An initial center point is set to be $\mathbf{x}_{c}^{i=0}=\mathbf{0}$, where $i$ is the iteration number. In order to start the search for the center points in an iterative manner, the global modes at the origin are assumed to be approximately the same over the uncertainty space in STEP 1.

STEP 2: At the initial center point, $\mathbf{x}_{c}^{i=0}=\mathbf{0}$, which is the origin of the uncertainty space, the first-order approximation of the diagonalized ROM is made as follows

$$
\overline{\mathbf{Z}}=(\overline{\mathbf{Z}})_{\mathbf{x}_{c}^{i=0}}+\sum_{k=1}^{n}\left(\frac{\partial \overline{\mathbf{Z}}}{\partial x_{k}}\right)_{\mathbf{x}_{c}^{i=0}}\left(x_{k}-x_{c, k}^{i=0}\right)
$$

In order to apply the finite difference method, the $\mathbf{Z}^{R O M}$ matrices are constructed at the perturbed points as well as at the center point, and the diagonal terms of the perturbed $\mathbf{Z}^{R O M}$ matrices are obtained with the global modes at the center point $\mathbf{x}_{c}^{i=0}=\mathbf{0}$. 


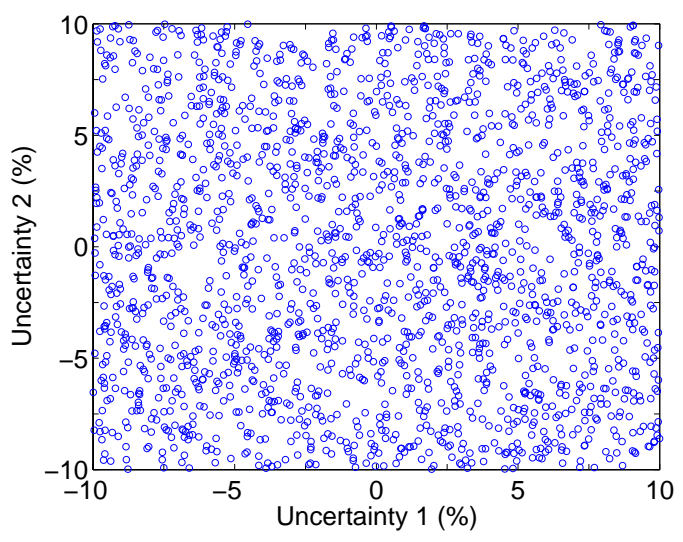

Figure 5. The sampling points that were generated in order to search for center points

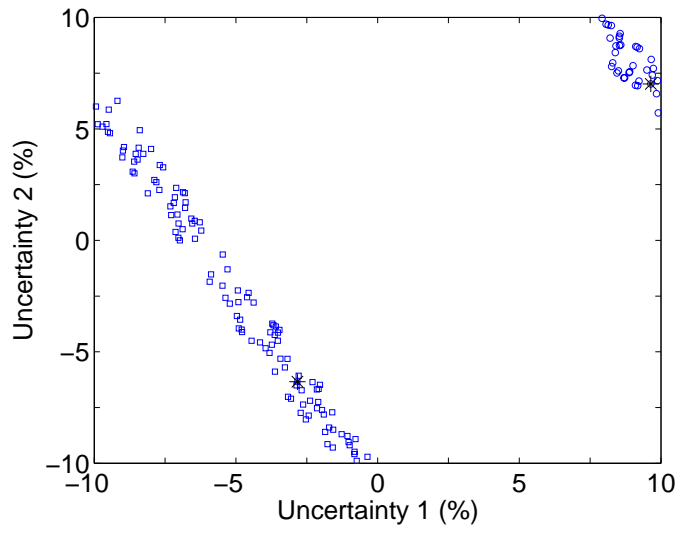

Figure 6. At iteration $i=1$, the subsets $S_{m=31}^{i=1}$ and $S_{m=32}^{i=1}$ and the newly identified center points (marked with stars) are shown

STEP 3: With the first-order approximation of Eq. (28), the subset, $S_{m}^{i=1}$, should be identified. $S_{m}^{i=1}$ is defined to have a relatively low magnitude of the imaginary part of the impedance for mode number $m$ such that

$$
S_{m}^{i=1}=\left\{\mathbf{x}_{r}\left|D_{0} \leq\right| \operatorname{Im}\left(\bar{z}_{m}\left(\mathbf{x}_{r}\right)\right) \mid \leq D_{\alpha} \quad \text { and } \quad \mathbf{x}_{r} \in S\right\}
$$

where $D_{\alpha}$ is defined as $\alpha$ percentile of $D$, and $D$ is defined as follows

$$
D=\left\{y\left|y=\min _{m}\right| \operatorname{Im}\left(\bar{z}_{m}\left(\mathbf{x}_{r}\right)\right) \mid \quad \text { and } \quad \mathbf{x}_{r} \in S\right\}
$$

Thus, $D_{0}$ in Eq. (29) is the lowest value in the set of $D$, which can be expressed as

$$
D_{0}=\min _{\mathbf{x}_{r} \in S} \min _{m}\left|\operatorname{Im}\left(\bar{z}_{m}\left(\mathbf{x}_{r}\right)\right)\right|
$$

Furthermore, based on each identified subset $S_{m}^{i=1}$, a new center point $\mathbf{x}_{c, m}^{i=1}$ associated with a specific mode number $m$ should also be identified with the following minimization problem

$$
\min _{\mathbf{x}_{r} \in S_{m}^{i=1}}\left|\operatorname{Im}\left(\bar{z}_{m}\left(\mathbf{x}_{r}\right)\right)\right|
$$

such that its solution $\mathbf{x}_{r}$ is an updated center $\mathbf{x}_{c, m}^{i}$.

For example, in Fig. 6, the subsets $S_{m=31}^{i=1}$ and $S_{m=32}^{i=1}$ are obtained using Eqs. (29)-(31), where $\alpha$ is specified as 7.5\%. In addition, the newly identified center points from each $S_{m}^{i=1}$, using Eq. (32), are marked with stars. As shown in this example case, there can be a few center points over the uncertainty space, rather than just one.

STEP 4: With each newly identified center point, $\mathbf{x}_{c, m}^{i}$, the first-order approximation of the diagonalized ROM is made as STEP 2

$$
\overline{\mathbf{Z}}=(\overline{\mathbf{Z}})_{\mathbf{x}_{c, m}^{i}}+\sum_{k=1}^{n}\left(\frac{\partial \overline{\mathbf{Z}}}{\partial x_{k}}\right)_{\mathbf{x}_{c, m}^{i}}\left(x_{k}-x_{c, m, k}^{i}\right)
$$

with the global modes at each center point $\mathbf{x}_{c, m}^{i}$.

STEP 5: In the same manner as STEP 3, with the updated first-order approximation Eq. (33), the modal coordinate $m$, the subset $S_{m}^{i+1}$, and the corresponding hyperplane $\operatorname{Im}\left(\bar{z}_{m}\right)=0$ should be updated. First, $S_{m}^{i+1}$ can be described with Eq. (33) as

$$
S_{m}^{i+1}=\left\{\mathbf{x}_{r}\left|D_{0} \leq\right| \operatorname{Im}\left(\bar{z}_{m}\left(\mathbf{x}_{r}\right)\right) \mid \leq D_{\alpha} \quad \text { and } \quad \mathbf{x}_{r} \in S\right\}
$$

The hyperplane $\operatorname{Im}\left(\bar{z}_{m}\right)=0$ that is close to the current center point $\mathbf{x}_{c, m}^{i}$, about which the first-order approximation Eq. (33) is obtained, and the corresponding subset $S_{m}^{i+1}$ are identified and kept. Other subsets and hyperplanes, which are farther from the current center point $\mathbf{x}_{c, m}^{i}$, are discarded. 


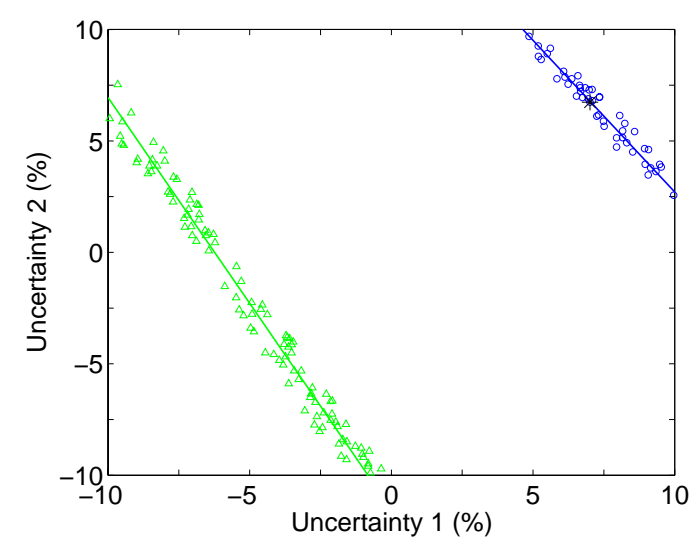

Figure 7. For iteration 2, mode 31, the subset that is closer to the center point $\mathbf{x}_{c, m=31}^{i=1}=(9.64,7.02)$ is kept (marked with circles), and the other subset is discarded

With the updated subset $S_{m}^{i+1}$ in Eq. (34), the center point $\mathbf{x}_{c, m}^{i+1}$ should also be updated with the following minimization problem

$$
\min _{\mathbf{x}_{r} \in S_{m}^{i+1}}\left|\operatorname{Im}\left(\bar{z}_{m}\left(\mathbf{x}_{r}\right)\right)\right|
$$

such that its solution $\mathbf{x}_{r}$ is an updated center $\mathbf{x}_{c, m}^{i+1}$.

For example, in Fig. 7, two hyperplanes are shown over the uncertainty range of interest through the first-order approximation constructed at the center point $(9.64,7.02)$ at the iteration $i=1$. The dark hyperplane is identified to be the closest to the center $(9.64,7.02)$, so the dark hyperplane and its subset $S_{m}^{i+1}$ are kept in Fig. 7. However, the light hyperplane shown in Fig. 7 is discarded. Furthermore, with Eqs. (34)-(35), the center point is updated and marked with a star in Fig. 7. Likewise, the other subset and center point are also updated in Fig. 8 .

Steps 4 and 5 are repeated, until convergence is achieved for the vibration response at a center point $\mathbf{x}_{c, m}^{i}$.

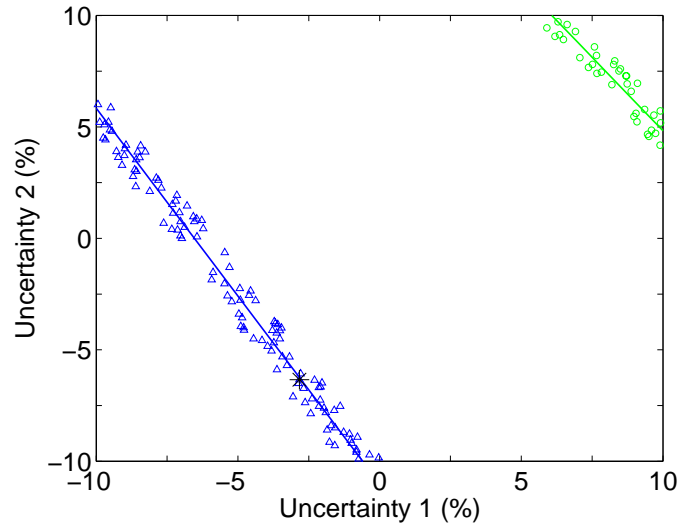

Figure 8. For iteration 2, mode 32, the subset that is closer to the center point $\mathbf{x}_{c, m=32}^{i=1}=(-2.83,-6.34)$ is kept (marked with triangles), and the other subset is discarded

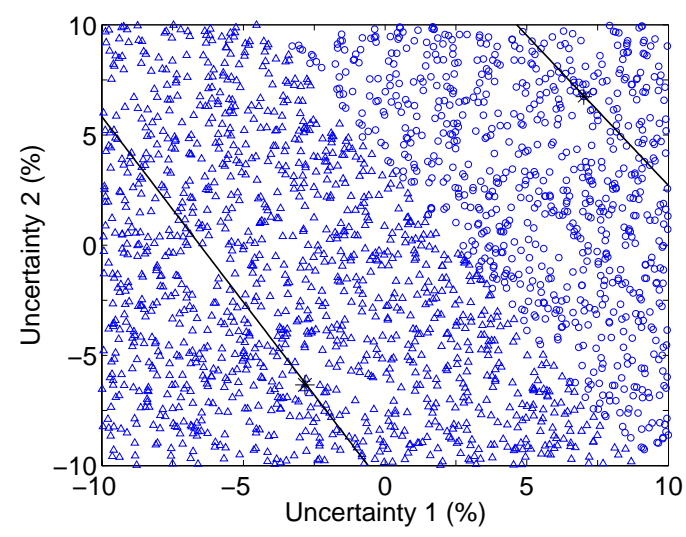

Figure 9. MCS sampling points divided into two groups (marked with circles and triangles) based on the closest identified hyperplane

STEP 6: With the identified center points and the corresponding hyperplanes, the sampling points for the Monte Carlo simulation (MCS), which are generated using appropriate distributions for the uncertainties, can be grouped to be close to each hyperplane identified in STEP 5. Thus, with the different first-order approximations constructed at each center point, the MCS can be performed. For example, in Fig. 9, the MCS sampling points are grouped into two subsets based on the distances from the identified dark hyperplanes in Figs. 7-8. Then, the MCS is performed with two first-order approximations of the diagonalized ROM, which are constructed at two different center points.

A Monte Carlo simulation (MCS) employing the procedures described in STEPs 1-6 is called a modebased MCS in this paper. This process yields the statistical properties of the vibration response, such as a probability distribution of the response. When it is desired to predict only the upper bound of the vibration response, some procedures of the mode-based MCS, more specifically STEPs 1-5, can provide the approximate upper bound without the MCS results, which is called an iterative maximum search method. It should also be noted that the accuracy of the mode-based MCS increases when the uncertainty space has fewer component-mode interactions. 


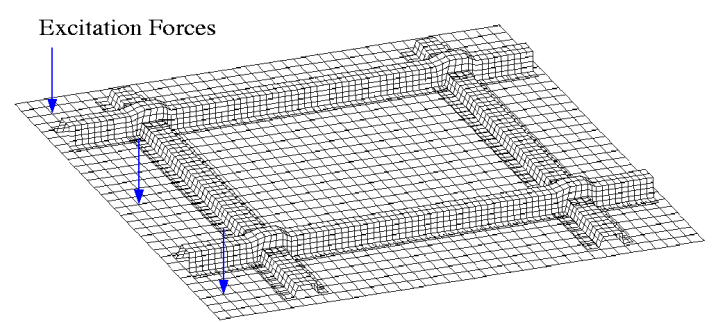

Figure 10. FE mesh for example model 2

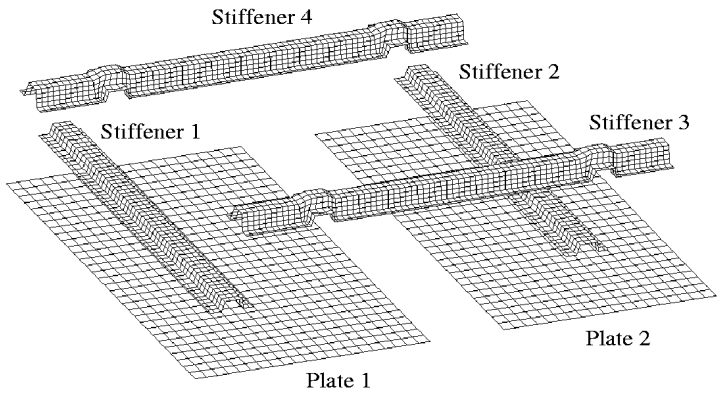

Figure 11. Substructures of example model 2

\section{B. Example Model 2: Stiffened Plate}

The finite element (FE) model of the stiffened plate considered in this second example is shown in Fig. 10. It is composed of 29,664 DOF. Since the reduced-order modeling technique used here is component-based, the structure is divided into a set of substructures, which are also called component structures. The dimension of the stiffened plate is $1.20 \mathrm{~m} \times 0.91 \mathrm{~m}$ in Fig. 10. The FE model is partitioned into six substructures, as shown in Fig. 11. The material properties for all substructures are Young's modulus $E=200 \mathrm{GPa}$, Poisson's ratio $\nu=0.28$, density $\rho=7840 \mathrm{~kg} / \mathrm{m}^{3}$, and thickness $t=2.5 \mathrm{~mm}$. The effects of uncertain properties of the stiffeners are considered. In particular, the thickness and Young's modulus are taken to be random variables, and two different cases are investigated.

In the first case, two random variables are considered: one for the thickness of the four stiffeners, and one for Young's modulus of the four stiffeners. They are assumed

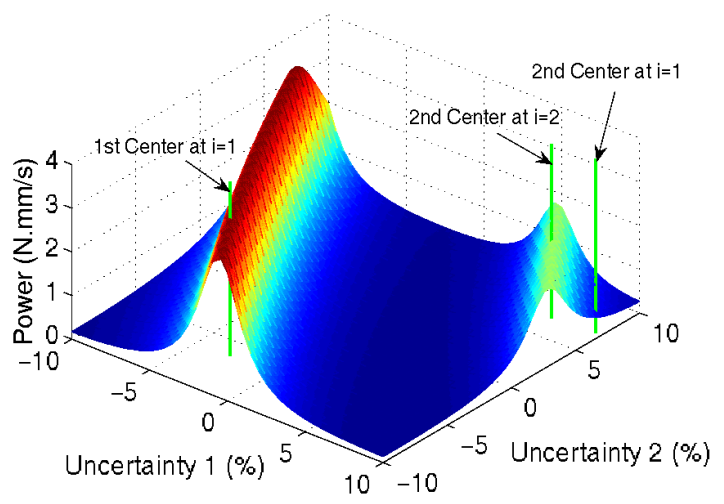

Figure 12. Center points from the mode-based MCS iteration process shown with the response surface constructed with exact ROMs to have $\pm 10 \%$ variation about the nominal values, with two independent, uniform probability density functions. Figures 5-9 show the processes of identification of the center points at the frequency of $228.79 \mathrm{~Hz}$. Table 2 shows the comparison of the CPU times. It is seen that the proposed mode-based MCS method is highly efficient. Figure 12 shows the iteration step at each center point, as well as the response surface constructed with $41 \times 41$ sampling points, which have equal interval. The center points at each iteration step and the power flow at the corresponding points are summarized in Table 3 for the stiffened plate with 2 uncertainties. Thus, the approximate maximum value over an uncertainty space at $228.79 \mathrm{~Hz}$ is $3.25(\mathrm{~N} . \mathrm{mm} / \mathrm{s})$ from the Table 3 . This is the result of the iterative maximum search, which is the sub-procedure of the mode-based MCS. Figure 13 depicts the cumulative

Table 2. Computational cost of MCS with 2,000 realizations for example model 2 at $228.79 \mathrm{~Hz}$

\begin{tabular}{|c|r|}
\hline Method & CPU Time \\
\hline Reference: MCS with exact ROMs & $3847 \mathrm{~min}$ \\
Method 1: Nominal-ROM-Based MCS & $760 \mathrm{~min}$ \\
Method 2: Mode-Based MCS & $48 \mathrm{~min}$ \\
\hline
\end{tabular}

probability obtained from the MCS with 2,000 exact ROMs and from the mode-based MCS with 2,000 approximate ROMs. Excellent agreement can be seen for the CDF results. Figure 14 shows the comparison of the CDF from the MCS with 2,000 exact ROMs and the MCS with 2,000 first-order approximations of diagonalized ROMs at the nominal point, not at the center points identified in an iterative manner as in the mode-based MCS method. With this comparison, the first-order approximation of the diagonalized ROM is 
Table 3. Iteration for 2 center points at $228.79 \mathrm{~Hz}$ for the stiffened plate with 2 uncertainties

\begin{tabular}{|c||c|c|}
\hline \multicolumn{1}{|c||}{ Iteration No. } & \multicolumn{2}{c|}{ Center points / Power Flow (N.mm/s) } \\
\hline \hline $\mathrm{i}=0$ & \multicolumn{2}{|c|}{ Nominal Point $(0,0)$} \\
\hline $\mathrm{i}=1$ & $(-2.83,-6.34) / 3.2521$ & $(9.64,7.02) / 0.4808$ \\
\hline $\mathrm{i}=2$ & Converged & $(7.01,6.74) / 1.7881$ \\
\hline $\mathrm{i}=3$ & & Converged \\
\hline
\end{tabular}

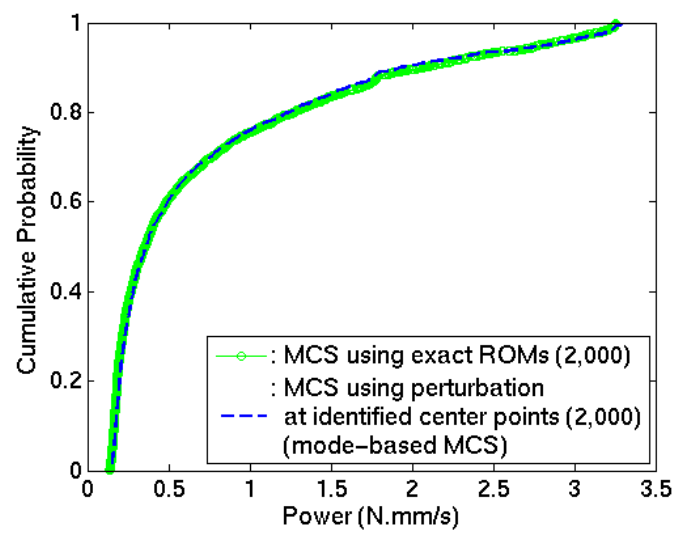

Figure 13. The cumulative probability from MCS using exact ROMs and MCS using first-order approximations of the diagonalized ROMs at the identified center points (mode-based MCS)

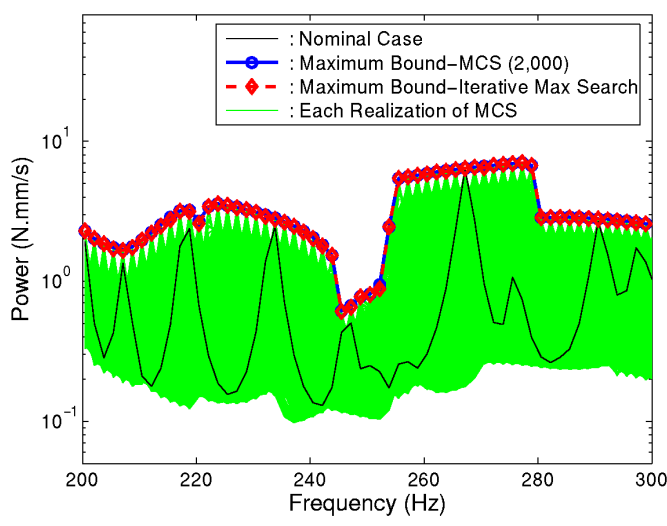

Figure 15. The upper bound of the power flow from MCS using exact ROMs and subprocedure of the mode-based MCS (iterative maximum search procedure)

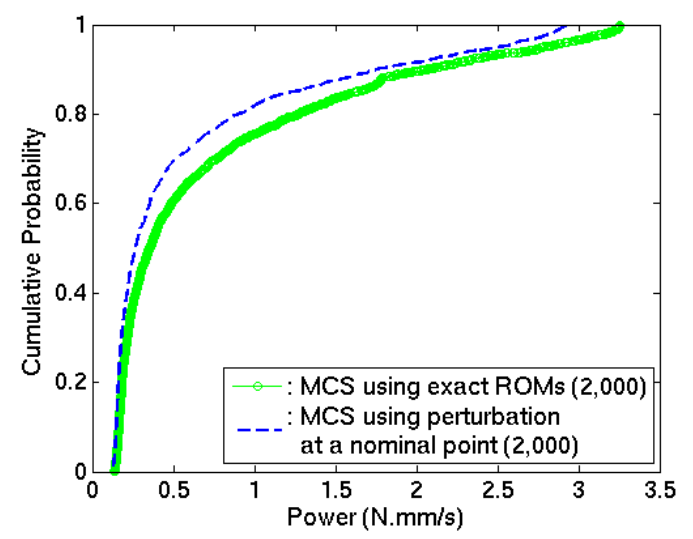

Figure 14. The cumulative probability from MCS using exact ROMs and MCS using a first-order approximation of the diagonalized ROM at a nominal point rather than at the center points

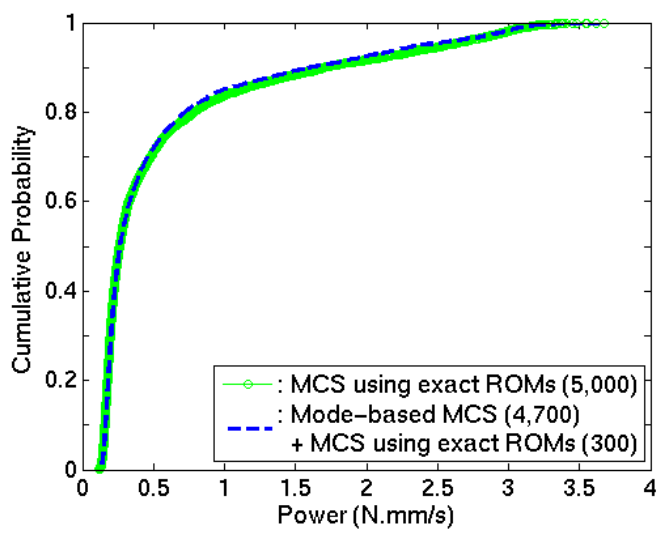

Figure 16. The cumulative probability from MCS using exact ROMs and enhanced mode-based MCS (mode-based MCS with 4,700 realizations and standard MCS with 300 realizations)

more sensitive to the point about which the ROM system is perturbed and approximated. Therefore, the identification of the center points is crucial to the performance of the mode-based MCS method. Figure 15 shows the upper bound from the MCS with exact ROMs, as well as from the iterative maximum search method, which is a sub-procedure of the mode-based MCS.

In the second case for the stiffened plate, eight random variables are considered: the thickness and Young's modulus of 4 stiffeners are assumed to be independent uncertainties. They are also assumed to have $\pm 10 \%$ variation about the nominal values, with eight independent, uniform probability density functions. In this case, the mode-based MCS is shown to be enhanced with a few standard MCS realizations in the subsets 
$S_{m}^{i+1}$ to achieve more accuracy in the range of the high percentile vibration response. Figure 16 shows the comparison of CDF results from the MCS with exact ROMs and the mode-based MCS enhanced with some standard MCS realizations. In this case, 4,700 realizations were performed with mode-based MCS and 300 realizations were performed with the standard MCS.

\section{Conclusions}

In this paper, component-mode-based implementations of Monte Carlo simulation (MCS) were presented. First, a previously developed substructuring technique was employed to generate reduced-order models (ROMs) in terms of component modes, and vibration power flow among the components was considered. Then, for performing probabilistic vibration analysis of structures with parameter uncertainties, MCS with two approximations of the ROM were examined: (1) MCS with a first-order approximation of the full ROM, and (2) MCS with first-order approximations of the diagonalized ROMs at the identified center points of the uncertainty range.

For the first MCS approach, called the nominal-ROM-based MCS method, the structure is partitioned into components such that each parameter uncertainty (or design variable) of interest can be expressed as a perturbation of component-level matrices. By doing so, this method can be applied even for the case where complicated component-mode interactions occur over an uncertainty space, and it provides more accurate predictions of the probability distribution of the vibration response or power flow. As a numerical example, a reinforced L-shaped plate with two parameter uncertainties was considered, and it was shown that the statistical properties were well predicted.

When there are fewer component-mode interactions over an uncertainty space, the second MCS approach, called a mode-based MCS method, can achieve greater efficiency. In this method, first-order diagonalized ROMs are constructed at the identified center points, and statistical properties such as the CDF can be predicted. In addition, an iterative maximum search procedure can be used to provide a rough approximation of the maximum response. This second MCS approach was applied to a stiffened plate structure, and the efficiency of the method was illustrated. It was also shown that the mode-based MCS method could be enhanced with additional standard MCS realizations for the sampling points in the subsets $S_{m}^{i+1}$ in order to obtain accurate predictions of high-percentile vibration response for the stiffened plate with eight uncertainties.

Overall, the first MCS approach is more general and has a wider range of application. However, when the component-mode interactions are not very complicated, the second MCS approach can be used to perform probabilistic vibration analysis with greater efficiency.

\section{Acknowledgment}

This research was supported by the Automotive Research Center, a U.S. Army Center of Excellence for modeling and simulation of ground vehicles, led by the University of Michigan.

\section{References}

\footnotetext{
${ }^{1}$ Craig, R. R. and Bampton, M. C. C., "Coupling of Substructures for Dynamic Analyses," AIAA Journal, Vol. 6, No. 7, 1968, pp. 1313-1319.

${ }^{2}$ Craig, R. R. and Chang, C.-J., "Substructure Coupling for Dynamic Analysis and Testing," Tech. Rep. NASA CR-2781, National Aeronautics and Space Administration, Washington, DC, February 1977.

${ }^{3}$ Brahmi, K., Bouhaddi, N., and Fillod, R., "Reduction of the Junction Degrees of Freedom Before Assembly in Dynamic Substructuring," Acoustics, Vibrations, and Rotating Machines, Vol. DE-84-2, American Society of Mechanical Engineers, New York, 1995, pp. 699-708.

${ }^{4}$ Aoyama, Y. and Yagawa, G., "Component Mode Synthesis for Large-Scale Structural Eigenanalysis," Computers and Structures, Vol. 79, No. 6, 2001, pp. 605-615.

${ }^{5}$ Castanier, M. P., Tan, Y.-C., and Pierre, C., "Characteristic Constraint Modes for Component Mode Synthesis," AIAA Journal, Vol. 39, No. 6, 2001, pp. 1182-1187.

${ }^{6}$ Rajashekhar, M. R. and Ellingwood, B. R., "A New Look at The Response Surface Approach For Reliability Analysis," Structural Safety, Vol. 12, 1993, pp. 205-220.

${ }^{7}$ Faravelli, L., "Response-Surface Approach for Reliability Analysis," Journal of Engineering Mechanics, Vol. 115, 1989, pp. 2763-2781.

${ }^{8}$ Romstad, K. M., Hutchinson, J. R., and Runge, K. H., "Design Parameter Variation and Structural Response," International Journal for Numerical Methods in Engineering, Vol. 5, 1973, pp. 337-349.
} 
${ }^{9}$ Benaroya, H. and Rehak, M., "Finite Element Methods in Probabilistic Structural Analysis: A Selective Review," Applied Mechanics Review, Vol. 41, 1988, pp. 201-213.

${ }^{10}$ Ang, A. H.-S. and Tang, W. H., Probability Concepts in Engineering Planning and Design, John Wiley \& Sons, New York, NY, 1975.

${ }^{11}$ Morris, M. D. and Mitchell, T. J., "Exploratory Design for Computational Experiments," Journal of Statistical Planning and Inference, Vol. 43, 1995, pp. 381-402.

${ }^{12}$ Ye, K. Q., Li, W., and Sudjianto, A., "Algorithmic Construction of Optimal Symmetric Latin Hypercube Design," Journal of Statistical Planning and Inference, Vol. 90, 2000, pp. 145-159.

${ }^{13} \mathrm{Wu}$, Y.-T., Millwater, H. R., and Cruse, T. A., "Advanced Probabilistic Structural Analysis Method for Implicit Performance Functions," AIAA Journal, Vol. 28, No. 9, 1990, pp. 1663-1669.

${ }^{14} \mathrm{Wu}$, Y.-T., Burnside, O. H., and Cruse, T. A., Computational Mechanics of Probabilistic and Reliability Analysis, chap. 7. Probabilistic Methods for Structural Response Analysis, Elmepress International, Lausanne, Switzerland, 1989 , pp. $181-195$.

${ }^{15}$ Youn, B. D., Choi, K. K., and Park, Y. H., "Hybrid Analysis Method for Reliability-Based Design Optimization," Journal of Mechanical Design, Vol. 125, 2003, pp. 221-232.

${ }^{16}$ Lee, S.-Y., Castanier, M. P., and Pierre, C., "A Most Probable Point-Based Method for Probabilistic Vibration Analysis of Structures with Uncertainties," Structural Dynamics: EURODYN 2005 (Proceedings of the 6th International Conference on Structural Dynamics, Paris, France, 4-7 September 2005), Vol. 2, Millpress, Rotterdam, The Netherlands, 2005, pp. 987-992.

${ }^{17}$ Lee, S.-Y., Efficient Probabilistic Vibration Analysis of Complex Structures Using Substructuring and Reliability Techniques, Ph.D. thesis, University of Michigan, Ann Arbor, 2006.

${ }^{18}$ Melchers, R. E., "Importance Sampling in Structural Systems," Structural Safety, Vol. 6, 1989, pp. 3-10.

${ }^{19}$ Harbitz, A., "An Efficient Sampling Method for Probability of Failure Calculation," Structural Safety, Vol. 3, 1986, pp. 109-115.

${ }^{20} \mathrm{Wu}$, Y.-T., "Computational Methods for Efficient Structural Reliability and Reliability Sensitivity Analysis," AIAA Journal, Vol. 32, No. 8, 1994, pp. 1717-1723

${ }^{21}$ Lee, S.-Y., Castanier, M. P., and Pierre, C., "Statistical Approximations of Power Flow for a Vehicle Structure with Uncertainties," Proceedings of the ASME Design Engineering Technical Conferences, Vol. 5B, American Society of Mechanical Engineers, New York, 2003, pp. 1305-1317.

${ }^{22}$ Tan, Y.-C., Castanier, M. P., and Pierre, C., "Characteristic-Mode-Based Component Mode Synthesis For Power Flow Analysis in Complex Structures," Collection of Technical Papers - AIAA/ASME/ASCE/AHS/ASC Structures, Structural Dynamics and Materials Conference, Vol. 1, AIAA, Reston, VA, 2000, pp. 908-917.

${ }^{23}$ Tan, Y.-C., Lee, S.-Y., Castanier, M. P., and Pierre, C., "Efficient Component-Based Vibration and Power Flow Analysis of a Vehicle Structure," ASME Paper IMECE2001/NCA-23509, Proceedings of the 2001 ASME International Mechanical Engineering Congress and Exposition, New York, 2001.

${ }^{24}$ Tan, Y.-C., Castanier, M. P., and Pierre, C., "Power Flow Analysis of Complex Structures Using Characteristic Constraint Modes," AIAA Journal, Vol. 43, No. 6, 2005, pp. 1360-1370. 